\title{
Composition followed by differentiation between weighted Bergman spaces and weighted Banach spaces of holomorphic functions
}

\author{
Elke Wolf \\ University of Paderborn \\ Mathematical Institute \\ D-33095 Paderborn, Germany \\ email: lichte@math. uni-paderborn. de
}

\begin{abstract}
Let $\phi$ be an analytic self-map of the open unit disk $\mathbb{D}$ in the complex plane. Such a map induces through composition a linear composition operator $C_{\phi}: f \mapsto f \circ \phi$. We are interested in the combination of $\mathrm{C}_{\phi}$ with the differentiation operator $\mathrm{D}$, that is in the operator $\mathrm{DC}_{\phi}$ : $f \mapsto \phi^{\prime} \cdot(f \circ \phi)$ acting between weighted Bergman spaces and weighted Banach spaces of holomorphic functions.
\end{abstract}

\section{Introduction}

Let $\mathbb{D}$ denote the open unit disk in the complex plane. For an analytic self-map $\phi$ of $\mathbb{D}$ the classical composition operator $\mathrm{C}_{\phi}$ is given by

$$
\mathrm{C}_{\phi}: \mathrm{H}(\mathbb{D}) \rightarrow \mathrm{H}(\mathbb{D}), \mathrm{f} \mapsto \mathrm{f} \circ \phi,
$$

where $H(\mathbb{D})$ denotes the set of all analytic functions on $\mathbb{D}$. Combining this with differentiation we obtain the operator

$$
\mathrm{DC}_{\phi}: \mathrm{H}(\mathbb{D}) \rightarrow \mathrm{H}(\mathbb{D}), f \mapsto \phi^{\prime} \cdot\left(f^{\prime} \circ \phi\right) .
$$

2010 Mathematics Subject Classification: 47B33, 47B38

Key words and phrases: composition operator, differentiation operator, weighted Bergman spaces, weighted Banach spaces of holomorphic functions 
Composition operators occur naturally in various problems and therefore have been widely investigated. An overview of results in the classical setting of the Hardy spaces as well as an introduction to composition operators is given in the excellent monographs by Cowen and MacCluer (cf. [6]) and Shapiro (cf. [13]).

Next, let us explain the setting in which we are interested. Bounded and continuous functions $v: \mathbb{D} \rightarrow] 0, \infty[$ are called weights. For such a weight $v$ we define

$$
\mathrm{H}_{v}^{\infty}:=\left\{\mathrm{f} \in \mathrm{H}(\mathbb{D}) ;\|\mathrm{f}\|_{v}:=\sup _{z \in \mathbb{D}} v(z)|f(z)|<\infty\right\} .
$$

Since, endowed with the weighted sup-norm $\|\cdot\|_{v}$, this is a Banach space, we say that $\mathrm{H}_{v}^{\infty}$ is a weighted Banach space of holomorphic functions. These spaces arise naturally in several problems related to e.g. complex analysis, spectral theory, Fourier analysis, partial differential and convolution equations. Concrete examples may be found in [4]. Weighted Banach spaces of holomorphic functions have been studied deeply in [3], but also in [5] and [2].

The weighted Bergman space is defined to be the collection of all analytic functions $f \in H(\mathbb{D})$ such that

$$
A_{v, p}:=\left\{f \in H(\mathbb{D}) ;\|f\|_{v, p}:=\left(\int_{\mathbb{D}}|f(z)|^{p} v(z) d A(z)\right)^{\frac{1}{p}}<\infty\right\}, 1 \leq p<\infty
$$

where $d A(z)$ denotes the normalized area measure. The investigation of Bergman spaces has quite a long and rich history. An excellent introduction to Bergman spaces is given in [9].

In this article we characterize boundedness and compactness of operators $\mathrm{DC}_{\phi}: \mathrm{A}_{v, \mathrm{p}} \rightarrow \mathrm{H}_{w}^{\infty}$ in terms of the involved self-map $\phi$ and the weights $v$ and $w$.

\section{Basics}

We study weighted spaces generated by the following class of weights. Let $v$ be a holomorphic function on $\mathbb{D}$ that does not vanish and is strictly positive on $\left[0,1\right.$. Moreover, we assume that $\lim _{r \rightarrow 1} v(r)=0$. Then we define the weight $v$ in the following way

$$
v(z):=v\left(|z|^{2}\right) \text { for every } z \in \mathbb{D} .
$$

Examples include all the famous and popular weights, such as 
1. the standard weights $v(z)=\left(1-|z|^{2}\right)^{\alpha}, \alpha \geq 1$,

2. the logarithmic weights $v(z)=\left(1-\log \left(1-|z|^{2}\right)\right)^{\beta}, \beta>0$,

3. the exponential weights $v(z)=e^{-\frac{1}{\left(1-|z|^{2}\right)^{\alpha}}}, \alpha \geq 1$.

For a fixed point $a \in \mathbb{D}$, we introduce a function

$$
v_{\mathrm{a}}(z):=v(\overline{\mathrm{a}} z) \text { for every } z \in \mathbb{D} \text {. }
$$

Since $v$ is holomorphic on $\mathbb{D}$, so is the function $v_{\mathrm{a}}$. Moreover, in particular, we will often assume that there is a constant $C>0$ such that

$$
\sup _{a \in \mathbb{D}} \sup _{z \in \mathbb{D}} \frac{v(z)}{\left|v_{a}(z)\right|} \leq \mathrm{C} \text {. }
$$

In the sequel we analyze which role condition (2) plays in the zoo of conditions on weights. Lusky (cf. [12]) studied weights satisfying the following conditions (L1) and (L2) (renamed after the author) which are defined as follows

$$
\inf _{n \in \mathbb{N}} \frac{v\left(1-2^{-n-1}\right)}{v\left(1-2^{-n}\right)}>0 \text { and (L2) } \limsup _{n \rightarrow \infty} \frac{v\left(1-2^{-n-j}\right)}{v\left(1-2^{-n}\right)}<1 \text { for some } j \in \mathbb{N} \text {. }
$$

Actually, weights which enjoy both conditions (L1) and (L2) are normal weights in the sense of Shields and Williams (see [14]). Obviously, condition (2) is connected with condition (L2) in the following way. If we change (2) as follows

$$
\sup _{a \in \mathbb{D}} \sup _{z \in \mathbb{D}} \frac{v(z)}{\left|v_{a}(z)\right|}<1
$$

then (L2) is equivalent with (3), if we assume that $|v(z)| \geq v(|z|)$ for every $z \in \mathbb{D}$. To show this, let us first assume that (L2) holds. Hence we can find $j \in \mathbb{N}$ such that

$$
\frac{v\left(1-2^{-n-j}\right)}{v\left(1-2^{-n}\right)}<1 \text { for every } n \in \mathbb{N} \text {. }
$$

Next, we fix $z \in \mathbb{D}$ and $a \in \mathbb{D}$. Then we can find $n \in \mathbb{N}$ such that

$$
|z| \geq 1-2^{-n-j} \text { and }|a z|<1-2^{-n}
$$

Now,

$$
\frac{v(z)}{|v(a z)|} \leq \frac{v\left(1-2^{-n-j}\right)}{v\left(1-2^{-n}\right)}<1 \text { for every } n \in \mathbb{N}
$$


Finally

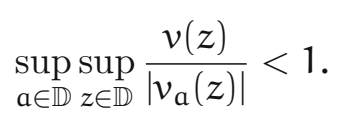

Conversely, we suppose that (3) is satisfied. We take $j=1$, fix $n \in \mathbb{N}$ and select

$$
a_{n}:=\frac{\left(1-2^{-n}\right)^{2}}{\left(1-2^{-n-1}\right)} .
$$

We obtain

$$
\frac{v\left(1-2^{-n-1}\right)}{v\left(1-2^{-n}\right)} \leq \frac{v(z)}{\left|v\left(a_{n} z\right)\right|} \leq \sup _{a \in \mathbb{D}} \sup _{z \in \mathbb{D}} \frac{v(z)}{\left|v_{a}(z)\right|}<1
$$

Thus, under some additional assumptions (2) is a weaker version of (L2). Calculations show that the standard weights as well as the logarithmic weights satisfy condition (2), while the exponential weights do not fulfill condition (2). Finally, we need some geometric data of the unit disk. A very important tool when dealing with operators such as defined above is the so-called pseudohyperbolic metric given by

$$
\rho(z, a):=\left|\sigma_{a}(z)\right|,
$$

where $\sigma_{\mathrm{a}}(z):=\frac{\mathrm{a}-z}{1-\overline{\mathrm{a}} z}, z, \mathrm{a} \in \mathbb{D}$, is the Möbius transformation which interchanges $a$ and 0 .

\section{Results}

Lemma 1 Let $v(z)=f(|z|)$ for every $z \in \mathbb{D}$, where $\mathrm{f} \in \mathrm{H}(\mathbb{D})$ is a function whose Taylor series (at 0 ) has nonnegative coefficients. We assume additionally that $v$ satisfies condition (2). Then there is a constant $\mathrm{C}>0$ such that

$$
|f(z)| \leq C^{\frac{1}{p}} \frac{\|f\|_{v, p}}{v(0)^{\frac{1}{p}}\left(1-|z|^{2}\right)^{\frac{2}{p}} v(z)^{\frac{1}{p}}} .
$$

Proof. Recall that a weight $v$ as defined above may be written as

$$
v(z):=\max \{|g(\lambda z)| ;|\lambda|=1\} \text { for every } z \in \mathbb{D} .
$$

We will write $g_{\lambda}(z):=g(\lambda z)$ for every $z \in \mathbb{D}$. Next, fix $\lambda \in \mathbb{C}$ with $|\lambda|=1$. Moreover, let $\alpha \in \mathbb{D}$ be an arbitrary point. We consider the map

$$
\mathrm{T}_{\alpha, \lambda}: A_{v}^{p} \rightarrow A_{v}^{p}, T_{\alpha, \lambda} f(z)=f\left(\sigma_{\alpha}(z)\right) \sigma_{\alpha}^{\prime}(z)^{\frac{2}{p}} g_{\lambda}\left(\sigma_{\alpha}(z)\right)^{\frac{1}{p}} .
$$


Then a change of variables yields

$$
\begin{aligned}
\left\|T_{\alpha, \lambda} f\right\|_{v, p}^{p} & =\left.\int_{\mathbb{D}} v(z)\left|f\left(\sigma_{\alpha}(z)\right)\right|\right|^{p}\left|\sigma_{\alpha}^{\prime}(z)\right|^{2}\left|g_{\lambda}\left(\sigma_{\alpha}(z)\right)\right| \mathrm{dA}(z) \\
& \leq \int_{\mathbb{D}}\left|f\left(\sigma_{\alpha}(z)\right)\right| \frac{v(z)}{v\left(\sigma_{\alpha}(z)\right)}\left|\sigma_{\alpha}^{\prime}(z)\right| \mathrm{dA}(z) \\
& \leq \mathrm{C} \int_{\mathbb{D}} \int_{\mathbb{D}}\left|\mathrm{f}\left(\sigma_{\alpha}(z)\right)\right| v\left(\sigma_{\alpha}(z)\right)\left|\sigma_{\alpha}^{\prime}(z)\right|^{2} \mathrm{dA}(z) \\
& \leq \mathrm{C} \int_{\mathbb{D}} v(\mathrm{t})|\mathrm{f}(\mathrm{t})|^{\mathrm{p}} \mathrm{dA}(\mathrm{t})=\mathrm{C}\|\mathrm{f}\|_{v, p}^{\mathrm{p}}
\end{aligned}
$$

Now put $h_{\lambda}(z):=T_{\alpha, \lambda}(z)$ for every $z \in \mathbb{D}$. By the mean value property we obtain

$$
v(0)\left|h_{\lambda}(0)\right|^{p} \leq \int_{\mathbb{D}} v(z)\left|h_{\lambda}(z)\right|^{p} \mathrm{dA}(z)=\left\|h_{\lambda}\right\|_{v, p}^{p} \leq c\|f\|_{v, p}^{p} .
$$

Hence

$$
v(0)\left|h_{\lambda}(0)\right|^{p}=v(0)|f(\alpha)|^{p}\left(1-|\alpha|^{2}\right)^{2}\left|g_{\lambda}(\alpha)\right| \leq C\|f\|_{\nu, p}^{p} .
$$

Since $\lambda$ was arbitrary we obtain that

$$
v(0)|f(\alpha)|^{p}\left(1-|\alpha|^{2}\right)^{2} v(\alpha) \leq C\|f\|_{v, p}^{p}
$$

Thus,

$$
|f(\alpha)| \leq C^{\frac{1}{p}} \frac{\|f\|_{v, p}}{v(0)^{\frac{1}{p}}\left(1-|\alpha|^{2}\right)^{\frac{2}{p}} v(\alpha)^{\frac{1}{p}}} .
$$

Since $\alpha$ was arbitrary, the claim follows.

Lemma 2 Let $v(z)=f(|z|)$ for every $z \in \mathbb{D}$, where $\mathrm{f} \in \mathrm{H}(\mathbb{D})$ is a function whose Taylor series (at 0) has nonnegative coefficients. We assume additionally that $v$ satisfies condition 2. Then for every $f \in A_{v}^{p}$ there is $C_{v}>0$ such that

$$
|\mathrm{f}(z)-\mathrm{f}(w)| \leq \mathrm{C}_{v}\|\mathrm{f}\|_{\nu, p} \max \left\{\frac{1}{\left(1-|z|^{2}\right)^{\frac{2}{p}} v(z)^{\frac{1}{p}}}, \frac{1}{\left(1-|w|^{2}\right)^{\frac{2}{p}} v(w)^{\frac{1}{p}}}\right\} \rho(z, w)
$$

for every $z, w \in \mathbb{D}$.

Proof. The proof is completely analogous to the proof given in [17]. Hence we omit it here. 
Lemma 3 Let $v(z)=f(|z|)$ for every $z \in \mathbb{D}$, where $\mathrm{f} \in \mathrm{H}(\mathbb{D})$ is a function whose Taylor series (at 0) has nonnegative coefficients. We assume additionally that $v$ satisfies condition (2). Then for $\mathrm{f} \in \mathrm{H}_{v}^{\infty}$ and $z \in \mathbb{D}$ :

$$
\left|f^{\prime}(z)\right| \leq \frac{M}{v(0)^{\frac{1}{p}}\left(1-|z|^{2}\right)^{1+\frac{2}{p}} v(z)^{\frac{1}{p}}}\|f\|_{v, p} .
$$

Proof. By Lemma 2 we have that

$$
|f(z)-f(w)| \leq \frac{M}{v(0)^{\frac{1}{p}}}\left\{\frac{1}{\left(1-|z|^{2}\right)^{\frac{2}{p}} v(z)^{\frac{1}{p}}}, \frac{1}{\left(1-|w|^{2}\right)^{\frac{2}{p}} \nu(w)^{\frac{1}{p}}}\right\} \rho(z, w)\|f\|_{v, p} .
$$

Now

$$
\begin{aligned}
& \left|\frac{f(z+h)-f(z)}{|h|}\right| \\
& \leq \frac{M}{v(0)^{\frac{1}{p}} h} \max \left\{\frac{1}{\left(1-|z+h|^{2}\right)^{\frac{2}{p}} v(z+h)^{\frac{1}{p}}}, \frac{1}{\left(1-|z|^{2}\right)^{\frac{2}{p}} v(z)^{\frac{1}{p}}}\right\} \rho(z+h, z)\|f\|_{v, p} \\
& =\frac{M}{v(0)^{\frac{1}{p}}|h|} \max \left\{\frac{1}{\left(1-|z+h|^{2}\right)^{\frac{2}{p}} v(z+h)^{\frac{1}{p}}}, \frac{1}{\left(1-|z|^{2}\right)^{\frac{2}{p}} v(z)^{\frac{1}{p}}}\right\} \\
& \mid \frac{z+h-z}{1-\overline{(z+h)} \mid\|f\|_{v, p}} \\
& =\frac{M}{v(0)^{\frac{1}{p}}} \max \left\{\frac{1}{\left(1-|z+h|^{2}\right)^{\frac{2}{p}} v(z+h)^{\frac{1}{p}}}, \frac{1}{\left(1-|z|^{2}\right)^{\frac{2}{p}} v(z)^{\frac{1}{p}}}\right\} \\
& \left|\frac{1}{1-\overline{(z+h) z}}\right|\|\mathrm{f}\|_{v, p} .
\end{aligned}
$$

Finally, let $h$ tend to zero and obtain

$$
\left|f^{\prime}(z)\right| \leq \frac{M}{v(0)^{\frac{1}{p}}\left(1-|z|^{2}\right)^{1+\frac{2}{p}} v(z)^{\frac{1}{p}}}\|f\|_{\nu, p} .
$$

Proposition $1 \operatorname{Let} v(z)=\mathrm{f}(|z|)$ for every $z \in \mathbb{D}$, wehre $\mathrm{f} \in \mathrm{H}(\mathbb{D})$ is a function whose Taylor series (at 0) has nonnegative coefficients. We assume additionally that $v$ satisfies condition (2). Then $\mathrm{DC}_{\phi}: \mathrm{A}_{v, p} \rightarrow \mathrm{H}_{w}^{\infty}$ is bounded if and 
only if

$$
\sup _{z \in \mathbb{D}} \frac{w(z)\left|\phi^{\prime}(z)\right|}{\left(1-|\phi(z)|^{2}\right)^{1+\frac{2}{p}} v(\phi(z))^{\frac{1}{p}}}<\infty .
$$

Proof. First, we assume that (4) is satisfied. Applying Lemma 1 we obtain

$$
\left\|D C_{\phi} f\right\|_{w}=\sup _{z \in \mathbb{D}} w(z)\left|\phi^{\prime}(z) \| f^{\prime}(\phi(z))\right| \leq C \sup _{z \in \mathbb{D}} \frac{w(z)\left|\phi^{\prime}(z)\right|}{\left(1-|\phi(z)|^{2}\right)^{1+\frac{2}{p}} v(\phi(z))^{\frac{1}{p}}} .
$$

Hence $\mathrm{DC}_{\phi}: \mathrm{A}_{v, \mathrm{p}} \rightarrow \mathrm{H}_{w}^{\infty}$ must be bounded.

Conversely, let $a \in \mathbb{D}$ be arbitrary. Then there exists $f_{a}^{p}$ in the unit ball of $H_{v}^{\infty}$ such that $\left|f_{a}(a)\right|^{p}=\frac{1}{\tilde{v}(a)}$. Now put

$$
g_{a}(z):=f_{a}(z) \varphi_{a}(z) \text { for every } z \in \mathbb{D} .
$$

Hence $\left\|g_{\mathrm{a}}\right\|_{v, p}^{\mathrm{p}}=\int_{\mathbb{D}}\left|g_{\mathrm{a}}(z)\right|^{\mathrm{p}} v(z) \mathrm{dA}(z) \leq \sup _{z \in \mathbb{D}} v(z)\left|\mathrm{f}_{\mathrm{a}}(z)\right|^{\mathrm{p}} \int_{\mathbb{D}}\left|\varphi_{\mathrm{a}}(z)\right|^{\mathrm{p}} \mathrm{dA}(z) \leq$ K. Moreover,

$$
g_{a}^{\prime}(z)=f_{a}^{\prime}(z) \varphi_{a}(z)+f_{a}(z) \varphi_{a}^{\prime}(z) \text { for every } z \in \mathbb{D} .
$$

Next, we assume that there is a sequence $\left(z_{n}\right)_{\mathfrak{n}} \subset \mathbb{D}$ such that $\left|\phi\left(z_{n}\right)\right| \rightarrow 1$ and

$$
\frac{w\left(z_{n}\right)\left|\phi^{\prime}\left(z_{n}\right)\right|}{\left(1-\left|\phi\left(z_{n}\right)\right|^{2}\right)^{1+\frac{2}{p}} v\left(\phi\left(z_{n}\right)\right)^{\frac{1}{p}}} \geq n \text { for every } n \in \mathbb{N} \text {. }
$$

Thus consider now $g_{\mathfrak{n}}(z):=g_{\phi\left(z_{n}\right)}(z)$ for every $n \in \mathbb{N}$ as defined above. Obviously $\left(g_{m}\right)_{n}$ is contained in the closed unit ball of $A_{v, p}$ and

$$
c \geq w\left(z_{n}\right)\left|\phi^{\prime}\left(z_{n}\right)\right|\left|g_{n}^{\prime}\left(\phi\left(z_{n}\right)\right)\right|=\frac{w\left(z_{n}\right)\left|\phi^{\prime}\left(z_{n}\right)\right|}{v\left(\phi\left(z_{n}\right)\right)^{\frac{1}{p}}\left(1-\left|\phi\left(z_{n}\right)\right|^{2}\right)^{1+\frac{2}{p}}} \geq n
$$

for every $n \in \mathbb{N}$ which is a contradiction.

Proposition 2 Let $v(z)=\mathrm{f}(|z|), z \in \mathbb{D}$, where $\mathrm{f} \in \mathrm{H}(\mathbb{D})$ is a function whose Taylor series (at 0) has nonnegative coefficients. Moreover, we assume that $v$ satisfies (2). Then the operator $\mathrm{DC}_{\phi}: \mathrm{A}_{v}^{\mathrm{p}} \rightarrow \mathrm{H}_{w}^{\infty}$ is compact if and only if

$$
\limsup _{|\phi(z)| \rightarrow 1} \frac{w(z)\left|\phi^{\prime}(z)\right|}{\left(1-|\phi(z)|^{2}\right)^{1+\frac{2}{p}} v(\phi(z))^{\frac{1}{p}}} .
$$


Proof. Let $\left(f_{n}\right)_{n}$ be a bounded sequence in $A_{v, p}$ that converges to zero uniformly on the compact subsets of $\mathbb{D}$. Let $M:=\sup _{\mathfrak{n}}\left\|f_{n}\right\|_{\nu, p}<\infty$. Given $\varepsilon>0$ there is $r>0$ such that if $|\phi(z)|>0$, then

$$
\frac{w(z)\left|\phi^{\prime}(z)\right|}{\left(1-|\phi(z)|^{2}\right)^{1+\frac{2}{p}} v(\phi(z))^{\frac{1}{p}}} \leq \frac{\varepsilon}{2 C_{v}} .
$$

On the other hand, since $f_{n} \rightarrow 0$ uniformly on $\{u ;|u| \leq r\}$, there is an $n_{0} \in \mathbb{N}$ such that if $|\phi(z)| \leq r$ and $n \geq n_{0}$, then $w(z)\left|f_{\mathfrak{n}}^{\prime}(\phi(z)) \| \phi^{\prime}(z)\right|<\frac{\varepsilon}{2}$. Now, an application of Lemma 3 yields

$$
\begin{aligned}
\left.\sup _{z \in \mathbb{D}} w(z) \mid f_{n}^{\prime}(\phi(z)) \| \phi^{\prime}(z)\right) \mid \leq & \sup _{|\phi(z)| \leq r} w(z)\left|f_{n}^{\prime}(\phi(z))\right|\left|\phi^{\prime}(z)\right| \\
& +\sup _{|\phi(z)|>r} w(z)\left|f_{n}^{\prime}(\phi(z))\right|\left|\phi^{\prime}(z)\right| \\
\leq & \frac{\varepsilon}{2}+\sup _{|\phi(z)|>r} \frac{C_{v} w(z)\left|\phi^{\prime}(z)\right|}{\left(1-|\phi(z)|^{2}\right)^{\frac{2}{p}+1} v(\phi(z))^{\frac{1}{p}}}<\varepsilon .
\end{aligned}
$$

Thus, the claim follows.

Conversely, we suppose that $\mathrm{DC}_{\phi}: \mathrm{A}_{v, \mathrm{p}} \rightarrow \mathrm{H}_{w}^{\infty}$ is compact and that there are $\delta>0$ and $\left(z_{n}\right)_{n} \subset \mathbb{D}$ with $\left|\phi\left(z_{n}\right)\right| \rightarrow 1$ such that

$$
\frac{w\left(z_{n}\right)\left|\phi^{\prime}\left(z_{n}\right)\right|}{\left(1-\left|\phi\left(z_{n}\right)\right|^{2}\right)^{1+\frac{2}{p}} v\left(\phi\left(z_{n}\right)\right)^{\frac{1}{p}}} \geq \delta .
$$

Since $\left|\phi\left(z_{n}\right)\right| \rightarrow 1$ there exist natural numbers $\alpha(n)$ with $\lim _{n \rightarrow \infty} \alpha(n)=\infty$ such that $\left|\phi\left(z_{n}\right)\right|^{\alpha(n)} \geq \frac{1}{2}$ for every $n \in \mathbb{N}$.

Next, for every $n \in \mathbb{N}$ we consider the function

$$
g_{\mathfrak{n}}(z):=f_{\mathfrak{n}}(z) \sigma_{\phi\left(z_{\mathfrak{n}}\right)}^{1+\frac{2}{p}}(z) z^{\alpha(n)}
$$

where $f_{\mathfrak{n}}^{p} \in H_{v}^{\infty}$ such that $\left\|f_{n}^{p}\right\|_{v} \leq 1$ and $\left|f_{n}\left(\phi\left(z_{\mathfrak{n}}\right)\right)\right|^{p}=\frac{1}{\tilde{v}\left(\phi\left(z_{\mathfrak{n}}\right)\right)}$. Then we obtain

$$
\begin{aligned}
\left\|D C_{\phi} f_{n}\right\|_{w} & \geq w\left(z_{n}\right)\left|\phi^{\prime}\left(z_{n}\right)\right| \mid f_{n}^{\prime}\left(\phi\left(z_{n}\right) \mid\right. \\
& \geq \frac{w\left(z_{n}\right)\left|\phi^{\prime}\left(z_{n}\right)\right|\left|\phi\left(z_{n}\right)\right|^{\alpha(n)}}{\tilde{v}\left(\phi\left(z_{n}\right)\right)^{\frac{1}{p}}\left(1-\left|\phi\left(z_{n}\right)\right|^{2}\right)^{1+\frac{2}{p}}} \\
& \geq \frac{1}{2} \frac{w\left(z_{n}\right)\left|\phi^{\prime}\left(z_{n}\right)\right|}{\tilde{v}\left(\phi\left(z_{n}\right)\right)^{\frac{1}{p}}\left(1-\left|\phi\left(z_{n}\right)\right|^{2}\right)^{1+\frac{2}{p}}} \geq \frac{1}{2} \delta .
\end{aligned}
$$

This is a contradiction. 


\section{References}

[1] J. M. Anderson, J. Duncan, Duals of Banach spaces of entire functions, Glasgow Math. J. 32 (1990), 215-220.

[2] K. D. Bierstedt, J. Bonet, A. Galbis, Weighted spaces of holomorphic functions on balanced domains, Michigan Math. J. 40 (2), 271-297.

[3] K. D. Bierstedt, J. Bonet, J. Taskinen, Associated weights and spaces of holomorphic functions, Studia Math. 127 (2) (1998), 137-168.

[4] K. D. Bierstedt, R. Meise, W. H. Summers, A projective description of weighted inductive limits, Trans. Am. Math. Soc. 272 (1) (1982), 107160 .

[5] K. D. Bierstedt, W. H. Summers, Biduals of weighted Banach spaces of holomorphic functions, J. Austral. Math. Soc. Ser. A. 54 (1993), 70-79.

[6] C. Cowen, B. MacCluer, Composition operators on spaces of analytic functions, CRC Press, Boca Raton, 1995.

[7] Z. Cučković, R. Zhao, Weighted composition operators on the Bergman space, J. London Math. Soc. (2) 70 (2004), 499-511.

[8] T. Domenig, Order bounded and p-summing composition operators, Studies on Composition Operators, Contemp. Math. 213, Amer. Math. Soc. Proyidence, RI, (1998), 27-41.

[9] P. Duren, A. Schuster, Bergman spaces, Mathematical Surveys and Monographs 100, Amer. Math. Soc, Providence, RI, (2004).

[10] R. Hibschweiler, Order bounded weighted composition operators, Banach spaces of analytic functions, Contemp. Math. 454, Amer. Math. Soc. Providence RI, (2008), 93-105.

[11] H. Hunziker, Kompositionsoperatoren auf klassischen Hardyräumen, Dissertation, Universität Zürich, 1989.

[12] W. Lusky, On the structure of $\mathrm{H} v_{0}(\mathrm{D})$ and $\mathrm{h} v_{0}(\mathrm{D})$, Math. Nachr. 159 (1992), 279-289.

[13] J. H. Shapiro, Composition operators and classical function theory, Springer, New York, Berlin, 1993. 
[14] A. L. Shields, D. L. Williams, Bounded porjections, duality and multipliers in spaces of harmonic functions, J. Reine Angew. Math. 299/300 (1978), 256-279.

[15] E. Wolf, Weighted composition operators between weighted Bergman spaces, RACSAM Rev. R. Acad. Cienc. Exactas Fis. nat. Ser A. Mat. 103 (1) (2009), 11-15.

[16] E. Wolf, Bounded, compact and Schatten class weighted composition operators between weighted Bergman spaces, preprint.

[17] E. Wolf, Differences of composition operators between weighted Bergman spaces and weighted Banach spaces of holomorphic functions, Glasgow Math. J. 52 (2) (2010), 325-332. 